\title{
LA AGRICULTURA SOCIAL (AS) COMO UNA OPORTUNIDAD EN EUROPA EN EL MARCO DE UN NUEVO ENFOQUE DE DESARROLLO RURAL SOSTENIBLE (DRS)
}

\author{
Antoni F. TULLA \\ Departamento de Geografía (Universidad Autònoma de Barcelona)
}

Antoni.tulla@uab.cat

\begin{abstract}
Resumen
El objetivo principal de esta presentación es mostrar como la AS resulta ser un actividad estratégica para el desarrollo local sostenible, tanto social, económica como ambiental, y como esta se implanta en un territorio concreto. La Agricultura social (AS) pretende facilitar la inserción laboral de colectivos en riesgo de exclusión social (RES) a través de la actividad agraria y su transformación o utilizando el contacto con la naturaleza. Podríamos definir la AS, a grandes rasgos, como un proceso de inclusión social y empoderamiento de colectivos en RES a través de la ocupación en actividades agrarias o en la transformación de sus productos mediante procesos artesanales, que permitan conectar al individuo con la naturaleza, el origen del producto, el ciclo de vida de los organismos vivos y con los beneficios de los procesos de manipulación manual. La ventaja comparativa que utiliza la segunda mejor opción (SMO) encuentra en la AS una actividad favorable a la creación del valor añadido en las áreas rurales periféricas. La metodología del retorno social de las inversiones (SROI) nos permite contabilizar cuál es el beneficio de la sociedad en relación al conjunto de las aportaciones de los colectivos de interés (stakeholders). Entonces hay que identificar los colectivos de interés implicados. Pretendemos analizar una selección de diez proyectos de AS sobre la base de datos de 161entidades en Cataluña. Para el conjunto hay un retorno social, económico y ambiental de aproximadamente $3 €$ por cada euro utilizado en el proyecto.
\end{abstract}

Palabras clave: Agricultura Social, Personas en riesgo de exclusión social, SROI, Tercer Sector Social, Valor añadido.

\begin{abstract}
The main objective of this presentation is to show how the Social Farming (SF) turns out to be a strategic activity for sustainable local development, both social, economic and environmental, and how it is implemented in a specific territory. The SF aims to facilitate the labor insertion of groups at risk of social exclusion (RSE) through agricultural activity and its transformation or using contact with nature. We could define the SF, broadly speaking, as a process of social inclusion and empowerment of groups in RSE through occupation in agricultural activities or in the transformation of their products through artisanal processes that allow connecting the individual with nature, the origin of the product, the life cycle of living organisms and the benefits of manual handling processes. The comparative advantage that the second best option (SBO) uses finds in the SF an activity favorable to the creation of added value in peripheral rural areas. The methodology of social return on investments (SROI) allows us to account for the benefit of society in relation to the total contributions of stakeholders. Then we must identify the stakeholders involved. We intend to analyze a selection of ten SF projects based on data from 161 entities in Catalonia. For the whole there is a social, economic and environmental return of approximately $€ 3$ for each euro used in the project.
\end{abstract}

Key words: Social Farming, People at risk of social exclusion, SROI, Third Social Sector, Added value. 


\section{Introducción: Agricultura Social (AS) y Desarrollo Rural Sostenible (DRS)}

Se pretende explicar el proceso crítico actual de la actividad en las áreas rurales en Europa. Primero se presenta la crisis económica, social y ambiental de las áreas en situación marginal con el auge del productivismo agrario y como, posteriormente se ha transformado el campo en un espacio multifuncional donde las actividades con valor añadido ayudan a su desarrollo y supervivencia. La agricultura social (AS), es una nueva actividad en esta diversificación del post-productivismo agrario. En muchos casos la AS puede ayudar no solo a la creación de empleo y a resolver la problemática de colectivos en riesgo de exclusión social (RES), sino también al desarrollo rural sostenible (DRS) de los territorios con predominio rural (GUIRADO, C. et al., 2014).

La AS pretende facilitar la inserción laboral de colectivos en RES a través de la actividad agraria y su transformación o utilizando el contacto con la naturaleza. Podríamos definir la AS, a grandes rasgos, como un proceso de inclusión social y empoderamiento de colectivos en RES a través de la ocupación en actividades agrarias o en la transformación de sus productos mediante procesos artesanales, que permitan conectar al individuo con la naturaleza, el origen del producto, el ciclo de vida de los organismos vivos y con los beneficios de los procesos de manipulación manual. Así pues, la AS es una actividad que añade nuevos significados a la práctica agrícola más allá de las cuestiones productivas, proporcionándole una función social concreta con el objetivo de aumentar la calidad de vida de las personas en RES. Los procesos de inclusión de estos colectivos a través de la AS se basan en la creación de lugares de trabajo o de formación, que a menudo van acompañados de un conjunto de medidas terapéuticas, de inserción laboral o de apoyo psicológico o sanitario. Las entidades promotoras y los equipos técnicos son, junto a las familias y las entidades del Tercer Sector Social (TSS) o la administración pública, sujetos básicos para el desarrollo con éxito de la AS (TULLA, A.F. et al., 2014).

\section{La transformación contemporánea del campo en Europa}

La agricultura comercial busca maximizar la eficiencia productiva desde mediados del Siglo XX. Lo cual ha comportado cambios profundos en explotaciones agrarias y áreas rurales en toda Europa. La agricultura intensiva de la denominada "Revolución Verde" ha convertido muchas áreas rurales en marginales, las cuales se han ido despoblando. La diversificación económica en las áreas rurales desde la década de 1980, incorporando las actividades turísticas y generando un valor añadido en los productos agrícolas, ha llevado a la actual multifuncionalidad (POTTER, C., 2004).

Al mismo tiempo, los consumidores aprecian cada vez más la agricultura orgánica y los productos de proximidad, adaptándose algunos de los agricultores a este nuevo tipo de mercado. Sin embargo, desde la crisis global iniciada el 2008, se ha agravado la situación económica de una gran parte de la población, que ha comportado:

- La importación de productos alimentarios de baja calidad, con una situación insostenible de elevados costes energéticos (transporte, productos químicos y maquinaria). 
TULLA, A., (2019). La agricultura social (AS) como una oportunidad en Europa en el marco de un nuevo enfoque de desarrollo rural sostenible (DRS). The Overarching Issues of the European Space- a strategic (re)positioning of environmental..., Porto, Fac.Letras Univ. Porto. pp. $387-407$ (DOI: 10.21747/9789898969149/agri)

- La falta de oportunidades de empleo que ha llevado a muchos jóvenes a buscar oportunidades económicas en el campo, dando lugar a experiencias innovadoras de producción, frecuentemente con valor añadido y criterios ecológicos, y distribuyendo la producción a través de circuitos cortos en mercados de proximidad.

Al final del siglo XX fue tomando mucha importancia la discusión sobre la "transición postproductivista" dando lugar a nuevas políticas para facilitar la diversificación productiva y la pluriactividad en las áreas rurales, inicialmente con el turismo y las actividades recreativas (EVANS, S. et al., 2002). En la Unión Europea (UE), fue creciendo la idea que era necesario evitar el impacto ambiental de la agricultura intensiva a través de las directrices de las agencias públicas. Se han facilitado ayudas a través de la "Política Agraria Comunitaria" (PAC) para la transición de las prácticas tradicionales hacia la agricultura ecológica u orgánica. Los agricultores han recibido subsidios para mejorar el medio ambiente plantando árboles, incrementando la ganadería extensiva y el uso de técnicas agrarias extensivas, y muy especialmente desarrollando productos agrarios de alta calidad. El resultado ha sido un cambio muy importante en la diversificación de actividades y de usos del suelo de las áreas rurales, que nos ha llevado a afirmar que hay una "naturaleza multi-funcional en el campo contemporáneo" junto a una mercantilización del campo (PERKINS, H.C., 2006).

Quizás nos convenga recordar los principales elementos de la Revolución Verde (1960-80):

- Uso intensivo de factores de producción, priorizando la producción por encima de las consideraciones sociales y medioambientales.

- La mecanización de la agricultura, el uso creciente de productos químicos, la reducción del factor trabajo, etc.

Lo cual ha comportado una distinción entre las áreas rurales de Europa, donde predomina:

- Agricultura productiva integrada en el mercado global con una protección decreciente de las políticas agrarias de la UE en los últimos 20 años.

- Áreas de agricultura productiva marginal, como las zonas de montañas, las regiones áridas o el periurbano, donde la población diversifica sus actividades, principalmente adaptándolas al turismo o a la producción agraria de calidad, con denominación de origen, consumo de proximidad y con productos de valor añadido, siguiendo las directrices de las políticas estructurales de las agencias de agricultura de la UE.

Un campo multifuncional significa que la actividad rural puede obtener resultados múltiples, no solo con la producción de alimentos y materias primas, sino también con productos agro-alimentarios de valor añadido, turismo, medio ambiente y beneficios sociales:

- La agricultura también puede contribuir a la sustentabilidad del paisaje rural, la protección de la biodiversidad, la creación de empleo junto con la diversificación de las actividades agroindustriales y los servicios y por lo tanto contribuir a la viabilidad de las áreas rurales (CUELLAR, M. et al., 2013).

- Esta nueva situación representa una "mercantilización del campo" (the commodification of the countryside), donde los consumidores que mayoritariamente viven en ciudades, están dispuestos a pagar más del valor de ciertos bienes. Un ejemplo sencillo es el coste 
TULLA, A., (2019). La agricultura social (AS) como una oportunidad en Europa en el marco de un nuevo enfoque de desarrollo rural sostenible (DRS). The Overarching Issues of the European Space- a strategic (re)positioning of environmental.... Porto, Fac. Letras Univ. Porto. pp. 387-407 (DOI: 10.21747/9789898969149/agri)

comparativo del suelo agrario, donde el precio se basa en el valor de su producción, mientras que si este suelo es ocupado por segundas residencias o actividades turísticas, entonces el precio depende de cuánto está dispuesto a pagar el consumidor. Entonces el uso agrario de este suelo puede estar fuera del mercado y, podemos hablar de un doble mercado sobre una parcela dependiendo del uso que se le quiera dar (BEST, 1989).

- La única solución es que los territorios implementen políticas de planificación para las actividades y los usos del suelo que se necesite, evitando el doble mercado. Algunas actividades rurales tradicionales como el ciclismo, la pesca, ir a caballo, ir en barca o caminar por la montaña se convierten en experiencias para vender a los turistas junto a la calidad de los productos agrarios, incorporando también las nuevas tecnologías.

\section{Desarrollo Rural, Sostenibilidad y Resiliencia}

Puede considerarse que hay un doble enfoque del concepto de desarrollo rural (DR): integración y transversalidad. EI DR trata con todas las dimensiones del mundo rural (especialización económica, patrimonio cultural, capital social y humano, medio ambiente, etc.). Pero al mismo tiempo se basa en el bienestar social y económico de la población, que se mide más por la calidad de vida en el campo que por el Producto Interior Bruto (PIB) per cápita del área.

EI DR, según la FAO/UNESCO (ATCHOARENA, D. y GASPERINI, L., 2004): comprende agricultura, educación, infraestructura, salud, fortalecimiento de las capacidades en función del empleo no agrícola, las instituciones rurales y las necesidades de los grupos vulnerables. El DR persigue la mejora de las condiciones de vida de la población rural, de manera equitativa y sostenible, tanto desde el punto de vista social como del medioambiente, gracias a un mejor acceso a los bienes (naturales, físicos, humanos, tecnológicos y al capital social) y servicios; y al control del capital productivo (en sus formas financiera o económica) que hacen posible mejorar su subsistencia de manera sostenible y equitativa.

EL DR debe combinar las políticas económicas, sociales y medioambientales con el objetivo que los recursos deben estar disponibles para las generaciones futuras y no agotarse en esta generación. En este sentido, hay una contraposición entre crecimiento, entendido únicamente como un incremento de las magnitudes económicas, y el desarrollo sostenible donde la calidad de vida, el uso correcto de los recursos y la protección ambiental son más importantes que el PIB de un área. EI "Desarrollo Sostenible" es un concepto formulado en el Informe Brundtland (Asamblea General de las UN, 1987) donde el desarrollo para satisfacer las necesidades actuales de la población no debería comprometer recursos que pueden ser necesarios para las generaciones futuras. Entonces se deberá evitar el uso excesivo de recursos escasos disponibles.

Desde la década de 1960, el DR intenta resolver, por lo menos, tres problemas. (1) Encontrar una base económica viable para las áreas rurales; (2) Conseguir un nivel de vida de cualidad para la población de estas áreas rurales, que incluya las condiciones medioambientales; y (3) Reorganizar la sociedad local rural en el marco de un mundo globalizado sin perder su identidad. Entonces, la resiliencia aparece como un proceso dinámico social, determinado parcialmente por la capacidad de 
las comunidades de actuar colectivamente y resolver los problemas en común (PLOEG, J.D. van, et al., 2000).

El concepto de "Resiliencia", incorporado hace menos de 20 años en las Ciencias Sociales y solo 10 en los análisis territoriales, puede también utilizarse en el DR. La noción de resiliencia aparece como la capacidad de un sistema ecológico-social para absorber las perturbaciones y reorganizarse mientras está sujeto a las fuerzas del cambio, siendo capaz de mantener lo básico de sus funciones, estructura, identidad y realimentación del sistema (KOCK EXTERCKOTER, R. et al., 2016).

Los ciclos económicos y las crisis forman parte de la vida diaria de las comunidades rurales. Los agricultores siempre deben encontrar soluciones para resolver acontecimientos inesperados del medio natural (granizo, heladas, sequía, etc.), así como de la incertidumbre económica y de los precios de mercado. Sin embargo, el incremento de la competencia, la globalización y los cambios medioambientales requiere cada vez más de respuestas muy ajustadas a la solución de los problemas concretos por parte de los grupos de agricultores y la administración.

Para poder captar mejor cuales son las políticas de la administración comentaremos dos actuaciones. Primera, como las ayudas estructurales de la UE (PAC), desde 1989, se han dirigido a las regiones rurales con tres objetivos principales: (1) Compensar los desequilibrios territoriales con un mejor acceso a las oportunidades económicas y sociales; (2) Corregir las desigualdades socioeconómicas, básicamente entre géneros y los grupos de edad; y (3) Protección medioambiental e implementación gradual de las políticas de sostenibilidad. Segunda, en la práctica significa "introducir nuevas políticas de DRS", como por ejemplo en la introducción de la metodología del Programa LEADER (Esparcia, J., 2000):

a) Fijar un diseño espacial de las políticas rurales en vez de un enfoque sectorial.

b) Toma de decisiones de abajo a arriba frente a las políticas de arriba abajo en las políticas económicas.

c) Sistemas participativos y de integración de los actores e instituciones en el territorio, debiendo remarcarse la creación de los "Grupos de Acción Local" (GAL) para coordinar estas políticas y acciones.

d) Acciones innovadoras para promover el DRS como los alimentos orgánicos, la energía agroeco, o la biomasa, con el apoyo científico y tecnológico así como de las finanzas públicas y privadas.

e) Análisis global de los problemas y oportunidades en un enfoque multi-sectorial.

f) Soporte financiero descentralizado a través de cooperativas, banca ética y gestión local

g) Una red organizativa de los proyectos, de sus actores e instituciones involucradas para poder mejorar la gestión con el intercambio de experiencias.

\section{Ventaja comparativa en las áreas rurales.}

El principio de ventaja comparativa, cuando se aplica utilizando el método de la segunda mejor opción (SMO), se fundamenta en dos supuestos: (1) El espacio es un recurso escaso que obliga a 
TULLA, A., (2019). La agricultura social (AS) como una oportunidad en Europa en el marco de un nuevo enfoque de desarrollo rural sostenible (DRS), The Overarching Issues of the European Space- a strategic (re)positioning of environmental.... Porto, Fac.Letras Univ. Porto. pp. $387-407$ (DOI: 10.21747/9789898969149/agri)

tomar decisiones para la mejor localización de actividades; y (2) Siempre existe una segunda localización, que sin ser la más óptima, permite localizar dichas actividades. Primero recordaremos de forma sintética la teoría de la ventaja comparativa y, segundo, presentaremos el método de la SMO (VERA, A. et al., 2011).

Cada región se especializa en producir bienes y servicios con ventaja comparativa en relación a otras regiones. Ventajas por los recursos naturales o por criterios de localización, así como por un mejor uso de factores intensivos o extensivos y de los recursos disponibles. El principio de ventaja comparativa de la escuela clásica nos dice que cada territorio se especializará en aquellos bienes y servicios que produce en mejores condiciones, en lugar de producir todos los bienes y servicios que necesita dicho territorio.

Para David Ricardo (1817), creador de la teoría de los costes comparativos entre distintas regiones, los costes se definen por las condiciones naturales y de desarrollo histórico para producir bienes. Ello genera rentas diferenciales derivadas de las diferentes calidades del suelo o de los recursos naturales disponibles. Henrich von Thünen (1826) incorpora los costes de localización y los costes de transporte al análisis anterior, añadiendo las rentas diferenciales de localización. En la aportación del modelo de Eli Heckscher y Bertil Ohlin (1933) se hace hincapié en las características de los factores de producción de cada región, tales como los niveles en tecnología y conocimiento de las regiones, introduciendo la consideración del valor añadido. De este modo, un territorio tenderá a exportar bienes y servicios que requieren factores intensivos de los que esté bien dotado mientras que procurará importar aquellos bienes y servicios que precisen factores intensivos de los que no disponga. Será Earling Olsen (1971) quien planteará que existe una ventaja relativa para cada región, que puede medirse con el coste de oportunidad de no especializarse en la producción de bienes y servicios para los que estuviera mejor dotada. Es decir, de no existir estos productos o servicios se debería importar.

Las regiones desarrolladas generan efectos negativos debido a un excesivo crecimiento y congestión. Estas deseconomías incrementan las desigualdades en el desarrollo, tal y como expuso Gunnar Myrdal (1957) con la teoría centro-periferia. Ante tales desigualdades, las regiones periféricas tienen la oportunidad de localizar actividades económicas de ventaja comparativa en relación a los grandes centros de producción y distribución, en una red de pequeñas y medianas empresas innovadoras, apoyadas por la lealtad espacial entre los agentes económicos y sociales de cada ámbito territorial (PALLARÈS-BARBERÀ et al., 2004). Peet y Hartwick (1999) constatan que los modelos de desarrollo cada vez se alejan más del simple crecimiento de las magnitudes económicas y priorizan, especialmente en las áreas periféricas, los pequeños emprendimientos basados en las actividades de valor añadido.

El dilema entre un desarrollo equilibrado y la polarización de un desarrollo regional desigual, sugiere la posibilidad que algunas regiones periféricas tengan "una ventaja comparativa relativa" tomando como referencia determinados bienes y servicios. Se han podido realizar proyectos de investigación en el Pirineo Catalán que demuestran como la transformación de productos lácteos, la ganadería ecológica extensiva para carne, las actividades turísticas respetuosas con el medio natural 
como la artesanía, el senderismo o el esquí de fondo, y también las manifestaciones culturales que se basan en la riqueza histórica, social y patrimonial del Pirineo.

Para ilustrar esta ventaja comparativa relativa en determinadas áreas del Pirineo Catalán explicaremos la transformación de productos lácteos. El espacio útil para los pastos del ganado vacuno es limitado y se deben tomar decisiones políticas en la planificación para poder preservarlo. Esta producción se lleva a cabo con la colaboración entre dos cooperativas. La Cooperativa Cadí, fundada en 1915, recoge la leche de vaca de sus socios y la transforma en mantequilla y quesos de alta calidad, que se exportan en más del 50\%. La Cooperativa Pirenaica, creada el 1944, abastece de factores de producción, especialmente alimentos para el ganado como el "Uni feed", combinación de piensos y minerales elaborados a las necesidades de cada explotación agraria que lo recibe "in situ" cada 2-3 días. Favorece desligar la producción de los prados y los campos de cada explotación, adquiriendo los productos que no aportan los socios. También facilita maquinaria, simientes y otros factores, pero lo más remarcable son los servicios como la centralización del cuidado de las "bravas" o la suplencia durante las vacaciones o las bajas médicas de los socios ganaderos a través de un equipo de trabajadores especializados contratados por la cooperativa.

En un estudio entre 1985 y 2015, la diferencia entre los precios percibidos por los aportantes a Cadí por $\mathrm{Kg}$ de leche y la media percibida por los ganaderos en España es de más del 18,06\%, y en relación a la media de los ganaderos en Francia de más del 10,53\%. Por parte de los costes, excluidos las factores tierra y trabajo, los precios medios pagados a Pirenaica son un $26,80 \%$ menos en relación a la media en España y un 10,78\% más en relación a la media en Francia. Lo cual implica una ventaja comparativa de un $44,86 \%$ en relación al conjunto de España y de la misma situación ($0,25 \%)$ que para la media en Francia. Al ser productos de calidad, con denominación de origen, los precios de venta son algo superiores a los productos de menor calidad pero tienen una buena aceptación en el mercado llegando a situaciones de escasez con la mantequilla y algún tipo de queso. En estos 30 años, la producción de $\mathrm{kg}$ de leche en Alt Urgell y Cerdanya (francesa y española) ha pasado de 71,3 a 94,8 millones, mientras que el número de explotaciones ha disminuido de 1.579 a 198. La producción media por explotación ha aumentado desde 45.176 a $478.889 \mathrm{~kg}$ de leche. El número de unidades de trabajo (UdT) ha pasado de 3.543 a solo 748 , aunque la media por explotación ha aumentado desde 2,24 a 3,78.

\section{La metodología de la Segunda Mejor Opción (SMO).}

El concepto de "segunda mejor opción" (SMO), formulada por Richard G. Lipsey y Kelvin Lancaster (1956), complementa el de la ventaja comparativa. Lo plantean como un equilibrio del "óptimo de Pareto" en el que solo falte una de las condiciones necesarias. En nuestra investigación tomamos la idea de que falte alguna condición para el desarrollo de actividades y servicios rentables en una región periférica. No hemos utilizado un análisis econométrico sino un análisis cualitativo (entrevistas en profundidad y grupos focales). 
Entonces la SMO se puede expresar como que "cada territorio puede desarrollar actividades o servicios, incluso si hay otros territorios mejor preparados para ello, cuando es la mejor especialización que se puede realizar en dicho territorio". La existencia de una SMO de un territorio significa impulsar con éxito una o más actividades económicas que permitan conseguir una ventaja comparativa. En las actividades con ventaja comparativa citadas anteriormente, hay un valor añadido importante que implica la transformación o elaboración de los productos con la incorporación de conocimiento, tecnología e innovación. Sin lugar a dudas, estas actividades se podrían realizar en otros territorios con mayor éxito pero son las mejores opciones que pueden desarrollarse en este territorio pirenaico con ventaja comparativa. Al decir que puede faltar alguna condición en cada caso para que sea óptimo, podría ser, por ejemplo, que el número de explotaciones para producir leche o carne es muy reducido para una producción de máxima rentabilidad.

En el método de la SMO (Figura 1), escogemos cuatro actividades o usos del suelo (filas) y tres regiones (columnas). Si asumimos que cada región debe tener por lo menos una de las actividades, entonces deberá acoger aquella para la que está más bien dotada (TULLA, A.F. et al., 2009). Se pueden utilizar diversos métodos para valorar los aspectos positivos y negativos de la localización en cada región, como puede ser la opinión de un grupo de expertos.

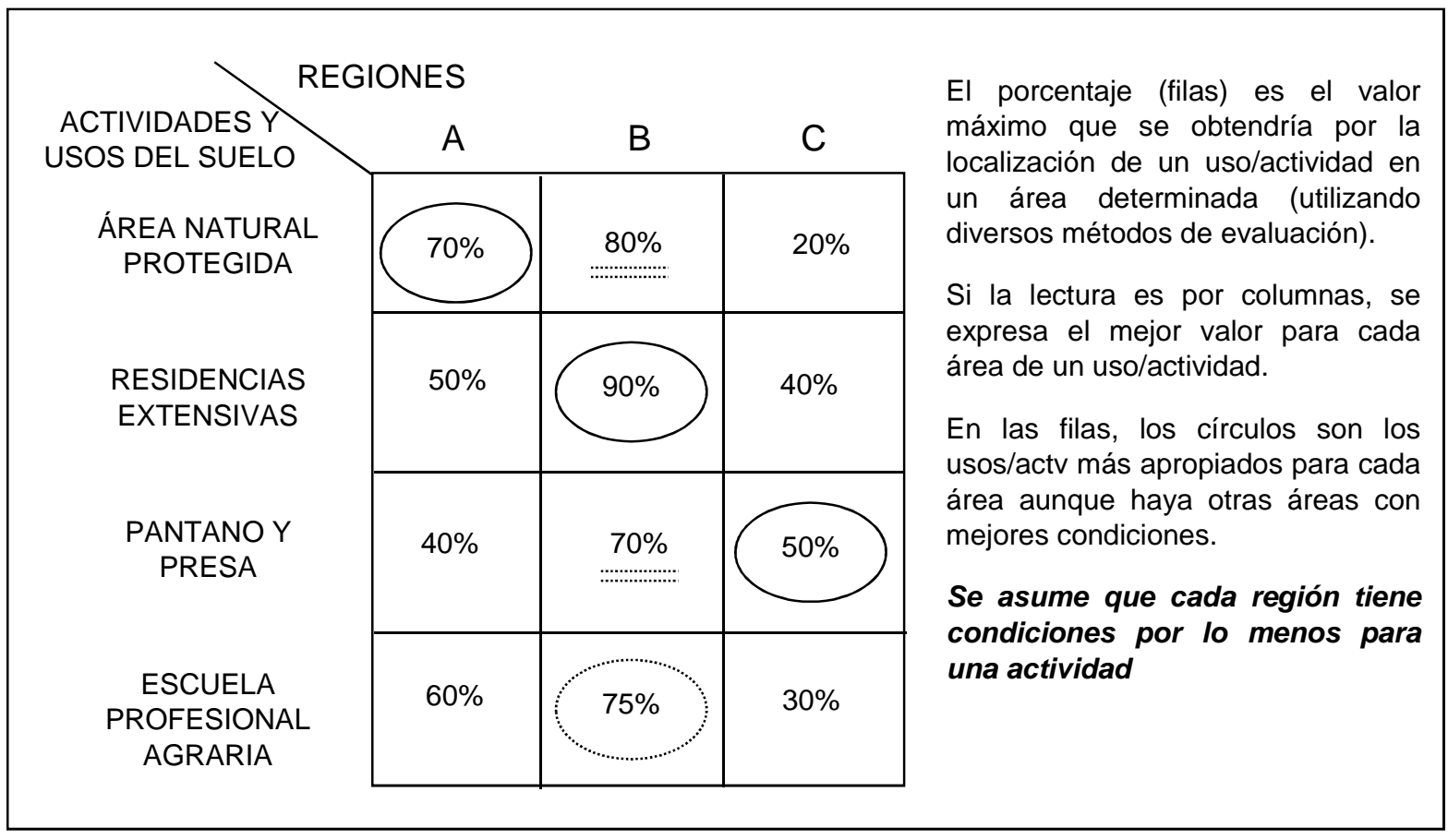

Figura 1- Ejemplo de aplicación del método de la Segunda Mejor Opción (SMO). Elaboración propia.

La región $\mathrm{B}$ tiene los valores máximos en los cuatro usos/actividades $\mathrm{y}$, por lo tanto, concentraría todas ellas mientras que las áreas A y B quedarían desiertas. La ventaja comparativa al hacer uso de la SMO buscará cual es la mejor capacitación de cada región para una actividad aunque pudiera haber otras regiones mejor dotadas. De esta forma, la región A acogerá el área natural (70\%) para la que ofrece mejores condiciones, la región $B$ las residencias (90\%) y la región C el pantano 
(50\%). Como cada región ya tiene una actividad o uso del suelo, entonces iniciaríamos una segunda ronda correspondiendo a la región B la Escuela Profesional (75\%). En nuestra investigación, apuntamos que la AS puede ser una SMO para muchas áreas rurales y periurbanas y puede considerarse de interés para el DRS.

\section{La AS como una oportunidad para el DRS.}

La agricultura social (AS) incluye un amplio abanico de actividades que tienen en común la práctica de producir, transformar o distribuir productos agrarios, con relación a la atención directa de colectivos y personas en riesgo de exclusión social (RES) por parte de entidades públicas o del Tercer Sector Social (TSS) básicamente.

La AS ofrece en el marco de la multifuncionalidad agraria:

- Ocupación y empoderamiento de las personas en RES.

- Terapias y servicios socio-sanitarios.

- Nuevas formas de producción y comercialización de productos agrarios.

- Una vinculación territorial (localidad, población, etc), institucional (especialmente con la administración local) y social (TSS, cooperativas, fundaciones, etc).

- Favorecer el desarrollo social sostenible, y

- Mejorar el medio ambiente y los paisajes rural y del periurbano.

Si nos fijamos en los elementos de la AS, los podemos definir de la forma siguiente:

(1) Actividad, productos agrarios y su transformación, servicios rurales, paisaje natural, rural y medioambiental;

(2) Objeto, la integración de personas o grupos en RES, facilitándoles servicios sanitarios y/o creando lugares de trabajo para obtener unos ingresos y empoderarlos al recuperar la confianza en si mismos;

(3) Sujetos, las diversas situaciones de personas en RES, con discapacidades (disminuciones mentales, físicas o psicológicas), con dificultades de integración económica y social e incluso en situación de riesgo de pobreza (desempleo estructural, adicciones -a drogas, por ejemplo-, presos, inmigrantes, etc.), y exclusión de jóvenes (por dificultades de aprendizaje o familias desestructuradas) o personas mayores (ingresos insuficientes o necesidades sociales);

(4) Recursos, servicios de salud o terapia, trabajo en explotaciones agrarias, públicos (ayudas y subvenciones, subsidios para los costes de la seguridad social o la vivienda, etc.) y privados (fundaciones, organizaciones religiosas, asociaciones y otras entidades del TSS que proveen de ayuda social o monetaria), acceso a la tierra y a los edificios, y el apoyo de la administración local, entre otros. El acceso a la tierra es muy complicado y hay organizaciones como "Terra Franca" en Cataluña que facilitan contactos entre personas interesadas en vender o alquilar y los proyectos de AS interesados. 
(5) Forma legal y tipos de gestión, hay diversas formas de organización y de condición legal de las entidades o proyectos de AS como las empresas privadas sin ánimo de lucro (algunas de carácter religioso), la administración (básicamente la local) y el TSS (cooperativas, asociaciones y los Centros Especiales de Trabajo -CET-).

(6) Las relaciones con y entre las instituciones, las redes de apoyo a plataformas de ayuda, creación y consolidación de la AS (Xarxa Agrosocial, por ejemplo) o diversas formas de colaboración entre proyectos de AS (asesoramiento, formación, comercio, compartir recursos, etc.).

La AS, como sector, se propone desarrollar proyectos en los que se combine en una relación circular: (a) La actividad agraria y su transformación y distribución, (b) La atención social, sanitaria y terapéutica, (c) Las políticas y programas sociales de la administración y el TSS, (d) La innovación social de productos orgánicos, con valor añadido y denominación de origen, (e) La inclusión de grupos socialmente vulnerables en RES, y (f) Un desarrollo local sostenible en colaboración con la administración local y otras actividades del territorio.

La implementación de la AS puede esquematizarse en dos aproximaciones, la conceptual en tres modelos (CARLOS GUIRADO y 9 autores más, 2017) y la institucional en cuatro modelos, según Francesco Di lacovo y Deirdre O'Connor (2009). Los modelos conceptuales tienen en cuenta la relación entre los usuarios de la AS con los gestores de los proyectos y, el uso de los elementos naturales (paisaje, plantas y animales) en las actividades realizadas:

a) El "Green Care" utiliza terapias que se fundamentan en el uso de la naturaleza para mejorar las condiciones de las personas en RES, especialmente las que tienen disminuciones mentales, físicas o psíquicas. Por lo general, los usuarios son tratados como clientes y pagan por este servicio, las familias directamente o a través de una subvención pública. Priorizan las atenciones terapéuticas de las personas en RES.

b) El "Social Farming" (Agricultura Social) ocupa personas en RES, que pueden recibir una remuneración junto a la atención social y sanitaria, e incluso pueden participar en el funcionamiento del proyecto. Uno de los objetivos es lograr el empoderamiento de estas personas. Los colectivos beneficiados son más amplios que en el Green Care. Las explotaciones agrarias priorizan la producción y el empleo de personas en RES.

c) El "Care Farming" se distinguiría por utilizar el trabajo agrario en explotaciones agrarias como terapia para mejorar la situación de personas con fragilidades. Tendría características de los otros dos modelos pero siempre utilizando explotaciones agrarias aunque sea con convenios con la administración para recibir subvenciones por el trabajo social y/o sanitario que llevan a cabo.

En cambio, los modelos institucionales toman en consideración la tipología de las entidades, los agentes implicados en el sector, el financiamiento de los proyectos, los modelos de gestión y el reconocimiento institucional de las iniciativas:

A) Modelo de la Sociedad del Bienestar (principalmente en los países nórdicos), donde hay un derecho fundamental de acceso a los servicios sociales y sanitarios a través de un sistema nacional de salud. La contribución tributaria de la población es alta. La AS se orienta más hacia los tratamientos terapéuticos (Green Care). 
TULLA, A., (2019). La agricultura social (AS) como una oportunidad en Europa en el marco de un nuevo enfoque de desarrollo rural sostenible (DRS), The Overarching Issues of the European Space- a strategic (re)positioning of environmental..., Porto, Fac Letras Univ. Porto. pp. $387-407$ (DOI: 10.21747/9789898969149/agri)

B) Modelo Corporativo (en los países de la Europa central), donde se garantiza el acceso a la salud pública con una combinación de los sistemas público y privado de los servicios sociosanitarios. Existe una normativa pública pero puede adjudicarse competencias a entidades privadas que lo gestionen. La AS se realiza en explotaciones agrarias que reciben subvenciones públicas previa formación de los responsables.

C) Modelo (neo) Liberal (Reino Unido e Irlanda, principalmente), donde la ayuda a las familias y a las personas en RES se realiza a través de entidades del TSS, el voluntariado y las instituciones religiosas (Charities). La AS está muy relacionada con proyectos sectoriales o con explotaciones agrarias.

D) Modelo Mixto (Países del sur de Europa), donde la reducción gradual del estado del bienestar frente a un auge del sector privado. Cada vez más ganan importancia las entidades del TSS y las acciones comunitarias de apoyo mutuo de las familias. El desarrollo de la AS se lleva a cabo en cooperativas, fundaciones y asociaciones con apoyos indirectos de la administración pública

Tal como se ha explicado los sistemas institucional y financiero relacionados con la AS varían según cada país en Europa. A título de ejemplo, en Italia, uno de los países pioneros donde la AS empezó en la década de 1970-79, encontramos organizaciones sin ánimo de lucro como las cooperativas sociales, iniciativas privadas y organizaciones de voluntariado, con ayuda pública o del TSS. En el 2000 había más de 6.000 cooperativas sociales y 190.000 empleados. En Francia, Les Jardins de Cocogne, del TSS, es una red de más de 120 huertos comunitarios, y existen redes desarrolladas por organizaciones agrarias para emplear personas en RES.

Hemos podido observar una cierta escasez de trabajos sobre la AS en España y Portugal, ya que hay pocos grupos de investigación que estén trabajando en esta temática. En España podemos destacar, en primer lugar, los trabajos realizados por el Equip de recerca en AS (ERAS-UAB) que ha estudiado esta actividad en Cataluña así como el retorno social de las inversiones, SROI (GUIRADO, C. et al., 2017), en segundo lugar, el Programa de Soberanía Alimentaria para la Lucha contra el Hambre y el Desarrollo Local impulsado por el Área de Cooperación y Solidaridad de la Universidad de Córdoba, que ha llevado a cabo un diagnóstico comarcal de las experiencias de AS en la campiña sur de esta ciudad (LAMARCA, 2015), y, en tercer lugar, las investigaciones del equipo de la Aliança per la Sobirania Alimentària de Cataluña, que analiza la práctica de la AS como un mecanismo para contribuir a la soberanía alimentaria y reducir la pobreza alimentaria de ciertos colectivos en Cataluña (Pomar, A. et al.,2015). También aparecen otras investigaciones y artículos de divulgación que han ido abordando el tema desde múltiples puntos de vista, como la sostenibilidad ecológica (GARCíALLORENTE et al., 2015), el Green Care (IRANZO-GARCIA et al, 2015) o los huertos comunitarios (FERNÁNDEZ, J.L. et al., 2011).

En el caso de Portugal, son conocidos los trabajos de Isabel de Maria Mourão y Luís Miguel Brito (2013), sobre las características y los beneficios de la horticultura social y terapéutica; de Ana Firmino (2011), sobre la situación de la AS en Portugal; y de Da Silva (2015), que realiza un estudio aplicado de los beneficios económicos, sociales y ambientales de los huertos sociales ecológicos de un municipio del norte de Portugal, cercano a Porto. Pese a que cada vez son más los investigadores 
y las instituciones que muestran interés por analizar este fenómeno social en auge, aún queda mucho por hacer en este campo. Algunos enfoques se aproximan al tema, a través del estudio de los espacios agrarios periurbanos (Matarán, 2013), la producción ecológica y la soberanía alimentaria (CUÉLLAR et al., 2013).

\section{Cataluña como un ejemplo de DRS a través de las estrategias de proyectos de AS.}

La AS surge en Cataluña entre 1974 y 1982 con dos iniciativas emblemáticas, l'Olivera y la Fageda, ambas cooperativas. El sector crece suavemente hasta la década de 1990 cuando hay un incremento significativo. Este momento coincide con un contexto socioeconómico que propicia la eclosión del voluntariado y una preocupación por los derechos de los colectivos con fragilidades que hasta este momento no tenían visibilidad social (MARBAN, V. et al., 2006). Los colectivos más usuales en la AS hasta este momento son los que tienen discapacidades o trastornos mentales. Después de la crisis de 1993, las administraciones públicas crearon programas de acción social, favoreciendo las ayudas a la AS. La crisis global de 2008 hizo aumentar la diversidad de los colectivos en RES, así como la mayor implicación del TSS al reducir las ayudas el sector público. Sin embargo, el número de proyectos crece, pasando de 58 (2008) a 206 (2017). De los cuales, 161 son propiamente de AS y los otros 45 únicamente de jardinería, que es un ámbito potencial para las iniciativas de AS.

El ámbito de actuación de estos 161 proyectos de AS, se focaliza en la inserción socio-laboral (46\%), para dar nuevas oportunidades de ocupación a las personas en RES, y en los huertos sociales (45\%), que es un fenómeno en expansión, promovidos por movimientos sociales, iniciativas ciudadanas o por la administración local, como respuesta a la precariedad y emergencia social como resultado de la crisis económica. Otras entidades trabajan con la terapia o la rehabilitación (5\%), orientada principalmente a personas con algún tipo de adicción, y un 4\% trabajan en el ámbito de la educación y la formación de jóvenes que han abandonado prematuramente los estudios.

La actividad principal de la AS es la agricultura (77\%), orientándose a la horticultura y los cultivos de la vid, los olivos y las setas. La transformación agroalimentaria (8\%) incluye la elaboración de mermeladas y conservas, cerveza artesana o transformación de lácteos. También hay trabajos forestales $(7 \%)$ y servicios $(6 \%)$ relacionados con la comercialización en circuitos cortos y con la restauración. Hay alguna experiencia en ganadería y artesanía. Cabe remarcar que en el $61 \%$ de los casos se produce con criterios ecológicos, fortaleciendo la imagen del sector pero también la atracción por parte de los consumidores responsables.

El colectivo principal como usuarios de la AS corresponde a las personas con discapacidad o trastorno mental (35\%) hasta la crisis de 2008. Después se estanca y, en cambio, aparecen nuevos colectivos que la situación de emergencia social ha puesto en evidencia. Sería el caso de los grupos en situación de pobreza material (28\%) o de desempleo (9\%). Estas personas tienen opciones de ocupación en el trabajo agrario, y también pueden cultivar sus propios productos de huerta en espacios cedidos. Las personas mayores (14\%) se han beneficiado de la creación de huertos para 
jubilados por parte de algunos ayuntamientos. Finalmente, otros colectivos socialmente vulnerables como los jóvenes, las mujeres maltratadas, las personas sin hogar, los inmigrantes o las personas del ámbito penitenciario, representan un $14 \%$.

En la forma jurídica de las entidades de AS predominan las organizaciones de carácter ciudadano y las que desarrollan su actividad sin ánimo de lucro, como las fundaciones (14\%) o las asociaciones (23\%). Hay instituciones privadas del TSS, con orientación mercantil (14\%) y las cooperativas $(12 \%)$ con una función social y asistencial. Finalmente, tenemos a las entidades promovidas desde la administración local (37\%), como los huertos sociales, que a menudo implican la colaboración entre el sector público y el privado, fomentando la cohesión social, la economía solidaria y el desarrollo local resiliente (Estela, O., 2015).

Las formas más usuales de organización legal son los "Centres Especials de Treball, CET" (31\%), empresas que garantizan el trabajo remunerado a personas con discapacidad y así garantizan su integración laboral en un entorno de trabajo protegido. Dependen del Departament d'Empresa i Coneixement de la Generalitat de Catalunya y están regulados por los Reales Decretos 2273/1985, de 4/12, y 1368/1985, de 17/07. Reciben subvenciones a los costes de la Seguridad Social y a los salarios. Las "Empresas de Inserción, El" (13\%), priorizan la integración socio-laboral de personas en RES con una tutela temporal de 2-3 años, preparándolas para el mercado laboral. Hay otras modalidades de inserción como los "Centros de Terapia Ocupacional, CO" $(2 \%)$ que promueven la incorporación de personas con dificultades al mercado laboral ordinario. En el contexto local, y a veces informalmente, aparecen acciones colectivas de soporte mutuo (42\%) para dar oportunidades a los colectivos en RES. Abarcan desde cooperativas agrícolas para fomentar la auto-ocupación de personas en paro estructural hasta la creación de huertos de autoconsumo promovidos por los Servicios sociales de las administraciones locales, sin ninguna figura jurídica específica coordinándose con otras entidades de AS para la atención social y la inserción laboral.

\section{Algunos ejemplos de la AS en Cataluña.}

Hemos estudiado a través de entrevistas en profundidad a 10 entidades de AS en Cataluña. Se han seleccionado de acuerdo con un criterio de ubicación y dependencia: 3 casos en el espacio periurbano metropolitano de Barcelona (L'Ortiga, Masia Can Calopa y l'Heura) con dependencia urbana; 3 casos en municipios rurales sin dependencia urbana (Casa Dalmases, Riu Verd y Aprodisca); y 4 casos en el ámbito rural pero con dependencia urbana de los promotores o de sus productos (La Klosca, Sambucus, Bolet ben fet y Delicies del Berguedà).

L'Ortiga (Can Montmany, Valldoreix, Sant Cugat del Vallès). Se orienta a la agro-ecología, la horticultura ecológica, la formación y los huertos sociales. Es un equipo técnico de 20 personas (35\% mujeres), con voluntarios y estudiantes en prácticas. Los usuarios son básicamente personas en riesgo de pobreza o desempleo, inmigrantes, discapacitados psíquicos y docentes interesados en la agricultura ecológica y la AS. Tienen 43 a tiempo completo con el salario mínimo y ayuda para la 
seguridad social. Se financia con la venta de la producción "en cestas" y a restaurantes. Tiene un convenio con el ayuntamiento de Sant Cugat de cesión de una finca por 20 años y por diversos programas sociales en el municipio.

Masia Can Calopa (Parc Natural de Collcerola, Barcelona). Es un proyecto fruto de un convenio entre el Ayuntamiento de Barcelona y la Cooperativa l'Olivera, por su know how, para recuperar una masia del siglo XVI y campos de viñedo en la Sierra de Collcerola. Producen vino y aceite de calidad, que lo utiliza el Ayuntamiento para sus eventos o l'Olivera lo comercializa. La masia acoge diversas actividades (Seminarios, cata de vinos) y gestionan una residencia para 17 jóvenes problemáticos que son el principal colectivo en RES. El equipo técnico son 12 (67\% mujeres), con diversos voluntarios. Se financia con la venta de vino y aceite embotellado y por el convenio con el Ayuntamiento y las ayudas públicas como Centro Especial de Trabajo (CET), a la seguridad social y formación de técnicos.

L'Heura (Horts de Can Salas, Terrassa). La entidad matriz "L'Heura SLL" es un CET dedicado a la jardinería, los viveros y el mantenimiento de los espacios verdes, orientado a la inserción sociolaboral de personas en RES. Desde la crisis de 2008 al reducirse las ayudas públicas se orientó a la producción de agricultura ecológica y a la formación. Son 6 técnicos (50\% mujeres) y 4 usuarios (25\% mujeres) con discapacidad psíquica o trastorno mental. Comercializan los productos propios y de otras explotaciones eco en una Agro-tienda, gestionan las cestas del grupo de consumo EcoMola y se benefician de las ayudas como CET.

Casa Dalmases (Cervera). Es una fundación sin ánimo de lucro que gestiona un palacete del siglo XVII fruto de una herencia. Produce cerveza y chocolate artesanos, y gestiona una tienda de productos sociales junto con la Asociación Alba. Es un equipo de 13 técnicos (46\% mujeres), la mayoría voluntarios y a tiempo parcial. Atienden un discapacitado intelectual a jornada completa y 8 (50\% mujeres) con dificultades especiales a tiempo parcial. Hay un patronato que hacen aportaciones regulares, se comercializa la producción y reciben ayuda pública y privada, tanto para el palacete como para las instalaciones productivas.

Riu Verd (Cal Robert, el Vinyet de Solsona). Es una cooperativa fundada el 2014 para inserir laboralmente y ofrecer educación a 6 jóvenes (33\% mujeres) problemáticos (familias desestructuradas o con perfil de delincuencia). Disponen de la masia, tierras e instalaciones (invernadero y obrador) de Cal Robert, donde cultivan huerta, hierbas aromáticas y elaboran productos alimentarios y conservas. Son 3 técnicos y se financian por la venta de la producción, las ayudas públicas de emprendimiento de la Generalitat de Catalunya y la entidad matriz (Asociación l'Afrau) como Programa de Garantía Juvenil.

Aprodisca Ambiental i Ecològica (Can Mas Fàbregas). Es una empresa de inserción (El) creada el 2015 y promovida por la Asociación Aprodisca (entidad matriz) fundada el 1986 en 
Montblanc para la integración social y laboral de los hijos de familias de la comarca. La El se orienta a la producción hortícola empleando 7 hombres en el paro de acuerdo con los Servicios Sociales de los municipios cercanos. La finca es una torre modernista en Constantí, cedida por 25 años por la Generalitat de Catalunya. Hay 3 técnicos (67\% mujeres) y voluntarios con dedicación variable. Se financia con la venta de la producción, la ayuda de la administración (estatal, autonómica y municipal), y la entidad matriz.

La Klosca (Sant Miquel de Mata, Mataró) es un CET (2009) promovido por la Asociación del Centro de Formación y Prevención (CFP) de 1970, dedicada a los viveros de plantas aromáticas y ornamentales. La Klosca se orienta al cultivo de huerta y la cría de gallinas para la producción de huevos ecológicos de proximidad. Los técnicos son una mujer y un hombre, y los usuarios son 8 personas (50\% mujeres) con trastorno mental severo. La finca con 1.772 gallinas y 2.250 docenes de huevos mensuales, se sitúa en el límite del Parc Natural del Montnegre-Corredor. Se financia con la venta de la producción, las ayudas públicas como CET, y las facilidades de crédito por parte de fundaciones privadas.

Sambucus (Manlleu) es un proyecto para dar oportunidades a personas con dificultades sociolaborales desde la crisis de 2008. Se constituye como cooperativa de trabajo y El con el apoyo de la administración local. Se fundamenta en la gestión del restaurante del Mercado Municipal de Manlleu, el cátering a colectivos, la producción de huerta, y el cultivo y secado de plantas aromáticas y medicinales con criterios ecológicos en la finca Mas Vinyoles en Sant Pere de Torelló. Hay 5 técnicos (60\% mujeres) y voluntarios, y 11 usuarios (45\% mujeres) de los Servicios Sociales municipales (mujeres inmigrantes, parados estructurales, jóvenes con problemas). Tienen subvenciones públicas como El, venden la producción y los servicios de restauración, pero también dependen del crédito de entidades del TSS.

Bolet ben fet (Sant Antoni de Vilamajor) es el resultado del proyecto de un emprendedor que producía setas artificiales (shitake y mitake) y se asoció como CET con la Cooperativa Taller Escuela de Barcelona (TEB) el 2007. Forma parte de TEB verde y producen en una granja en Sant Antoni de Vilamajor. Son 13 técnicos y voluntarios (38\% mujeres) para colaborar y atender 10 usuarios (20\% mujeres), con debilidad intelectual o jóvenes con riesgo de pobreza. La producción de setas, de calidad ecológica, se vende en restaurantes japoneses (un tercio se exporta al sur de Francia), y han recibido financiación del Programa Momentum del BBVA y como CET ayuda pública. Reducen gastos al formar parte de la administración de la entidad matriz, que les ayuda económicamente de forma puntual.

Portal Berguedà SL, con la marca Delicies del Berguedà (Cercs, Berga) es una El laboral promovida el 2011 por la Fundació Portal (FP) que da apoyo a los jóvenes con patología dual y a sus familias. La El produce yogurts naturales ecológicos con la leche de vaca de una granja próxima (Can Gris), que garantiza el bienestar animal y la calidad del producto. El equipo técnico son 3 hombres y 2 
voluntarios, mientras los usuarios son 6 jóvenes con patología dual que residen en pisos atendidos y se preparan para la inserción laboral. Se busca crear un producto de calidad con materias primas del territorio y orientado básicamente al consumo local y en el eje Berga-Manresa-Barcelona. El centro de producción recibe las ventajas económicas de la Agencia de desarrollo del Berguedà, y las subvenciones públicas como El. También se han acogido al Programa de Emprendimiento Social de Cataluña.

\section{El retorno a la sociedad (SROI) de la AS.}

El retorno de las inversiones a la sociedad (SROI) podemos definirlo como la medición de los valores sociales, medioambientales y económicos que se crean por una determinada acción de AS. Este método aparece el año 1997 cuando la Roberts Enterprise Development Fund (REDF), entidad del TSS que fomenta la inserción sociolaboral de personas en RES, quiso medir el impacto de empresas sociales en la ciudad de San Francisco (California, USA). En 2004 se creó The SROI Network, una red europea que pretende fijar "estándares" para medir el valor del retorno social a través de los cambios generados en los agentes de interés (Nicholls et al., 2012). El proceso de cálculo consta de 5 fases.

En la fase primera se define quienes participarán en el proceso y como lo harán. Se debe determinar los agentes de interés (stakeholders) y alcance temporal. Para cada uno de ellos se identifica y cuantifica la inversión realizada (inputs), la aportación a la entidad y los resultados (outputs) obtenidos. La inversión total será la suma de la inversión en euros que aporta cada agente de interés. Los resultados se refieren a las actividades en AS de la entidad, según la inversión realizada, el número de personas en RES atendidas, de voluntarios o de familiares, así como las acciones realizadas. A continuación explicaremos algunos de los stakeholders más frecuentes en la AS en Cataluña:

(a) Los/as trabajadores/as en RES, usuarios de las entidades de AS y que a menudo se benefician de un contrato de trabajo. Son el principal sujeto de los proyectos de AS.

(b) Los/as trabajadores/as profesionales (equipo técnico) con competencias agrarias, de atención social o económica, adquiridas antes o con la actividad realizada. Son los responsables del impulso y gestión del proyecto, como empresa productiva i social.

(c) Las personas voluntarias o en prácticas dan soporte al equipo técnico y mejoran el capital social. Adquieren habilidades, satisfacción y mejoran las relaciones sociales.

(d) Los familiares son el entorno más próximo a los/as usuarios/as, y experimentan cambios positivos como el bienestar emocional y material. Las entidades de AS dan soporte y descargan a los familiares de parte de la atención a personas en RES.

(e) Los clientes de la entidad son otras entidades o personas que valoran la calidad del producto y de los servicios que ofrecen. Aportan ingresos a la entidad de AS. 
TULLA, A., (2019). La agricultura social (AS) como una oportunidad en Europa en el marco de un nuevo enfoque de desarrollo rural sostenible (DRS), The Overarching Issues of the European Space- a strategic (re)positioning of environmental..., Porto, Fac. Letras Univ. Porto, pp. 387-407 (DOI: 10.21747/9789898969149/agri)

(f) Los proveedores son empresas que aportan inputs para la elaboración de los productos. Son agentes clave, ya que a menudo deben tener características específicas como las ecológicas o ser entidades sin ánimo de lucro.

(g) Las Asociaciones y Fundaciones son las promotoras de proyectos de AS, consideradas a veces como "entidades matriz", que proveen de financiamiento y otros apoyos.

(h) Las administraciones públicas dan subvenciones y ayudas para los lugares de trabajo protegidos y facilitan el desarrollo de la entidad. Es un stakeholder importante por registrar cambios importantes (recaudación de impuestos y desaparición del subsidio de empleo al crearse un lugar de trabajo) y aportar inversiones (Seguridad Social).

(i) La administración local es aún más importante por promover convenios con entidades de AS, al facilitar recursos a cambio de servicios como la atención a personas en RES.

(j) La comunidad y el territorio local donde se realiza la AS puede generar efectos positivos y generar cambios. La entidad de AS puede contribuir al desarrollo social y a la puesta en valor de los productos locales, así como generar sinergias con otras actividades del territorio. Puede ayudar a mejorar el medio ambiente y el paisaje.

En la segunda fase, se elabora el mapa del impacto. Este pretende ayudar a entender y articular la teoría del cambio para explicar de qué forma la organización crea valor mediante el uso de recursos a partir de determinar el valor de la aportación de cada stakeholder. Se deben poder identificar los cambios (outcomes) sociales, económicos y ambientales que son fruto de la actividad de la entidad para cada grupo de interés. El objetivo, en la AS, es la mejora de la autonomía personal de los usuarios en RES (ponderado por el número). Algunos de los cambios son difíciles de medir ya que requerirán estudios específicos. Como sería el caso de la valoración económica de una mejora de la biodiversidad o del riesgo de incendios.

En la tercera fase, se da un valor a los resultados (outputs). Para los cambios (outcomes) que hemos identificado se han establecido indicadores que explican cuál es el impacto que se ha conseguido y, además se cuantifican las unidades de cambio obtenidas por un período determinado. Se aplican aproximaciones monetarias específicas (financial proxy) para los indicadores, lo cual permite cuantificar los cambios conseguidos en unidades monetarias.

En la cuarta fase se mide el impacto total para cada uno de los cambios. Se cuantifica el impacto de la inversión que se relaciona con el valor de los resultados que se han obtenido y se corrigen los cambios (outcomes) restando las atribuciones que no han estado producidas por la entidad analizada a través de diversos correctores: peso muerto, atribución y deterioro, y también se aplica un corrector temporal por los cambios de más o menos de un año.

En la quinta fase, se realiza el cálculo del SROI. Se suman los beneficios, restando los elementos negativos, y se compara el resultado con la inversión. En el desarrollo de esta metodología es importante comunicar los resultados, transmitiendo toda la información a los stakeholders para poder contrastar y verificar los resultados obtenidos.

En el estudio realizado a nueve entidades de AS en Cataluña, la mediana aritmética del SROI ha sido de 3,02 € por cada euro invertido. La distribución de este retorno entre los stakeholders 
TULLA, A., (2019). La agricultura social (AS) como una oportunidad en Europa en el marco de un nuevo enfoque de desarrollo rural sostenible (DRS), The Overarching Issues of the European Space- a strategic (re)positioning of environmental.... Porto, Fac. Letras Univ. Porto. pp. 387-407 (DOI: 10.21747/9789898969149/agri)

podemos expresarlo en porcentajes: los usuarios $(16,6 \%)$, sus familias $(12,3 \%)$, el equipo técnico $(19,9 \%)$, el voluntariado $(3,3 \%)$, la administración pública $(21 \%)$, la Comunidad local y la región $(25,7 \%)$, y otros $(1,2 \%)$. Estos $3,02 €$ de retorno por cada euro invertido se distribuyen entre:

- Beneficios sociales como la inserción, facilitar servicios sanitarios o conseguir el apoderamiento de personas en RES.

- Beneficios económicos, como la ocupación remunerada de los colectivos en RES o la reducción de los gastos de la administración pública (subsidio de paro, recaudación de impuestos), además de los ingresos por la venta de los productos y servicios.

- Beneficios ambientales, como la recuperación de tierras de cultivo, la reducción de los riesgos de incendios, la reducción de las emisiones de gases contaminantes o la mejora de la biodiversidad.

\section{Resultados y Conclusiones de DRS.}

La agricultura intensiva de la denominada "Revolución Verde" ha marginado muchas áreas rurales que han terminado despoblándose. La diversificación económica en algunas de estas áreas desde la década de 1980, incorporando actividades turísticas y generando valor añadido en productos agrarios, ha permitido su recuperación parcial con la multifuncionalidad.

Los productos de valor añadido, como sería el caso de los productos lácteos de la Cooperativa del Cadí, con el soporte en la provisión de factores de producción de la Cooperativa Pirenaica, permiten afirmar que esta actividad puede considerarse como una ventaja comparativa en las regiones de montaña, utilizando el método de la segunda mejor opción (SMO)

La producción extensiva de ganado (y si es en orientación ecológica mejor) tiene una mejor relación con la calidad del medio ambiente y el paisaje que algunas de las producciones intensivas. Favorece la gestión de los recursos naturales y humanos en áreas de montaña como los Pirineos. Mejora la biodiversidad y hace disminuir los riesgos de incendios.

La mayor apreciación por parte de los consumidores de las ventajas de la agricultura orgánica y de proximidad ha favorecido la participación de los agricultores en los mercados locales. Al mismo tiempo, la menor oportunidad de empleo ha conllevado que los jóvenes busquen oportunidades en el campo con la nueva producción ecológica y de "circuito comercial corto".

La resiliencia aparece como un proceso social dinámico, determinado en parte por la capacidad de muchas comunidades de resolver problemas comunes y actuar colectivamente. En este sentido, los fondos estructurales de la PAC, desde 1989 han considerado tres objetivos básicos: Compensar los desequilibrios territoriales facilitando oportunidades económicas y sociales, Corregir las desigualdades económicas (género y edad, principalmente), y Proteger el Medio Ambiente implementando gradualmente políticas de sustentabilidad.

Las regiones periféricas, en base a la Teoría de la Ventaja Comparativa, pueden tener la oportunidad de nuevas localizaciones económicas por una congestión de las áreas centrales, pero también por la innovación y la lealtad espacial de las pequeñas y medianas empresas (PiMEs). 


\section{Resultados y Conclusiones de AS como un instrumento del DRS.}

Las actividades en las áreas periféricas, en el marco del DRS, se fundamentan más en los "costes relativos" por unidad que en los "costes totales". Por ello, es muy importante incorporar costes y beneficios sociales en el balance final. Entonces, vemos como la Agricultura Social (AS) aparece como una de las actividades como SMO en muchas regiones.

La AS muestra una expansión heterogénea en Europa, donde hay tradiciones y normas distintas entre los países del norte y centro en comparación con los del sur. Se ha elaborado una legislación específica en Italia, Bélgica y los Países Bajos. En Cataluña se ha pasado de 42 proyectos en el 2008 a 161 en el 2017, básicamente por la eclosión de los huertos sociales.

La AS en Cataluña aparece en la década de 1970 para atender las personas con dificultades especiales, básicamente disminuidos físicos, psíquicos o mentales, con asociaciones o fundaciones de origen familiar que reciben ayudas del Estado Español y/o la Generalitat de Catalunya. En el siglo XXI se han incrementado los colectivos en RES con mayores problemas económicos y sociales (personas en riesgo de pobreza o desempleo, con adicciones, etc.), y mayor implicación de las cooperativas.

Del estudio de la AS en Cataluña podemos considerar que hay 5 aportaciones importantes:

- Empoderamiento de personas socialmente vulnerables y recuperación de su dignidad.

- Contribución al desarrollo local y a la equidad territorial (resiliencia territorial)

- Fomentar la economía social y solidaria (ESS) y el cooperativismo.

- Difusión de proyectos sociales innovadores y de estrategias de promoción de la agricultura orgánica y de la producción agro-ecológica con mercados de proximidad,

- Contribución a la protección ambiental y la recuperación de suelo agrario.

Que se pueden resumir en los principios de equidad y justicia social. Muchas de las entidades de AS en Cataluña tienen una vocación ética y social, priorizando a las personas sobre los beneficios económicos. Se pretende dar la oportunidad de obtener un trabajo decente a los colectivos en RES, junto al uso de la terapia y otros servicios relacionados con la salud.

Los proyectos de AS, impulsados por entidades del Tercer Sector Social (TSS) o empresas sin ánimo de lucro, promueven el retorno a la sociedad (SROI) de beneficios sociales, económicos y ambientales (alrededor de $3 €$ por euro invertido en Cataluña). Estos proyectos buscan tener una cierta viabilidad económica sin renunciar a los objetivos sociales.

\section{Bibliografía}

ATCHOARENA, D.; GASPERINI, L., eds. (2004) Education for Rural Development. Towards New Policy Responses. FAO/UNESCO-IIEP, Roma.

BEST, S. (1989) The commodification of reality and the reality of commodification: Jean Baudrillard and post-modernism. Current Perspectives in Social Theory, 19: 23-51.

CUÉLLAR, M., CALLE, A. y GALLAR, D., eds. (2013) Procesos hacia la soberanía alimentaria. Perspectivas y prácticas desde la agroecología política. Barcelona, Ed. Icaria. 
DA SILVA, N. (2015): Estudo dos benefícios sociais, ambientais e económicos das hortas sociais biológicas do município da Póvoa de Lanhoso. Master dissertation. Instituto Politecnico do Viana do Castelo, Viana do Castelo.

DI IACOVO, F.; O'CONNOR, D. (2009) Supporting Policies for Social Farming in Europe. Progressing Multifunctionality in Responsive Rural Areas. So Far. Social Services in Multifunctional Farms ("Social Farming"). ARISA, Florencia.

ESPARCIA, J. (2000) The LEADER Programme and the Rise of Rural Development in Spain. Sociologia Ruralis, 40 (2): 200-207.

ESTELA, O. (2015) Politiche pubbliche per economie locali resilienti, Territorio della Riccerca su Insediamenti e Ambiente. Rivista Internazionale di cultura urbanistica, no 15: 81-94.

EVANS, S.; MORRIS, C.; WINTER, M. (2002) Conceptualizing agriculture: a critique of postproductivism as the new orthodoxy. Progree in Human Geography, 26: 313-32.

FERNÁNDEZ J.L. y MORÁN, N. (2011). «Huertos Comunitarios». El Ecologista, oํ 70, 23-26.

FIRMINO, A. (2011): Agricultura Social em Portugal - inovação de futuro incerto?, Comunicação oral no I Encontro de Agricultura Social, Escola Superior Agrária de Coimbra.

GARCíA-LLORENTE, M., ROSSIGNOLLI, C., MORUZZO, R. Y Di lacovo, F. (2015) «Social Farming practices to promote social-ecological sustainability in rural areas». Second International Conference on Agriculture in an Urbanizing Society, Rome (Italy) 14-17 September 2015.

GUIRADO, C.; BADIA, A.; TULLA, A.F.; VERA, A.; VALLDEPERAS, N. (2014) La agricultura social em Catalunya: innovación social y dinamización agroecológica para la ocupación de personas en riesgo de exclusión. Ager. Revista de estudios sobre despoblación y desarrollo rural, 17: 6597.

GUIRADO, C.; VALLDEPERAS, N.; TULLA, A. F.; SENDRA, L.; BADIA, A.; EVARD, C.; CEBOLLADA, A.; ESPLUGA, J.; PALLARÈS, I.; VERA, A. (2017). «Social Farming in Catalonia: rural local development, employment opportunities and empowerment for people at risk of social exclusion». Journal of Rural Studies, volume 56 (2017): 180-197.

INFORME BRUNDTLAND (1987) Informe de la Comisión Mundial sobre el Medio Ambiente y el Desarrollo. Asamblea General de las Naciones Unidas, 4-08-1987.

IRANZO-GARCÍA, E.; PASCUAL, J.A.; BLASCO, C. y FANSA, G. (2015). «Accessible landscapes and health: A proposal for a comprehensive development of the rural landscape and heritage resources for therapeutic purposes" en 23rd Annual Colloquium Sustainable Rural Systems: Smart Answers for a Smiling Future (Lisboa - Porto, 27th July - 2nd August, 2015).

KOCK EXTERCKOTER, R.; TULLA, A.F.; AZEVEDO DA SILVA, C. (2016) Análisis bibliométrico del concepto de resiliência aplicado al desarrollo regional. Documents d'Anàlisi Geogràfica, vol. 62 (2): 275-298.

LAMARCA, A. (2015): Agricultura Social: experiencias en la campiña sur de Córdoba, Área de Cooperación y Solidaridad (Universidad de Córdoba), Córdoba.

LIPSEY, R. G. and LANCASTER, K. (1956): The General Theory of Second Best. Review of Economic Studies, 24: 11-32.

MARBÁN, V.; RODRíGUEZ, G. (2006) Estado de bienestar y tercer sector social en España. EI estado de la investigación social. CIRIEC, Revista de economía pública, social y cooperativa, no 56: 117-139.

MATARÁN RUÍZ, A. (2013) «Participación social en la protección activa de los espacios agrarios periurbanos: un estado de la cuestión». Boletín de la Asociación de Geógrafos Españoles, no 63, 57-79.

MOURÃO, I. e BRITO, L.M. (2013) Horticultura Social e Terapêutica. Hortas urbanas e atividades com plantas no modo de produção biológico. Publindústria, Porto.

MYRDAL, G., (1957) Economic Theory and Under-Developed Regions. Gerald Duckworth \& Co.Ltd, London. 
NICHOLLS, J.; LAWLOR, E.; NEITZERT, E.; GOODSPEED, T.; CUPITT, S., eds. (2012) A Guide to Social Return on Investment. Office of The Third Sector. The SROI Network. Accounting for Value.

OHLIN, B., (1933) Interregional and International Trade. Harvard University Press, Cambridge, MA.

OLSEN, E. (1971): International Trade, Theory and Regional Income Differences. Amsterdam: NorthHolland.

PALLARÈS-BARBERÀ, M.; TULLA, A. F.; VERA, A. (2004) Spatial Loyalty and Territorial Embeddedness in the Multi-Sector Clustering of the Berguedà Region in Catalonia (Spain). Geoforum, 35(5): 635-649.

PERKINS, H.C. (2006) Commodification: re-resourcing rural áreas, 243-257, in Cloke, P.; Marsden, T. \& Mooney, P. (eds) Handbook of Rural Studies. Sage, London.

PLOEG, J.; RENTING, H.; BRUNORI, G.; KNICKEL, K.; MANNION, J.; MARSDEN, T.; DE ROEST, K.; SEVILLA-GUZMÁN, E.; VENTURA, F. (2000) Rural development: from practices and policies towards theory. Sociologia Ruralis, 40 (4): 391-408.

POMAR, A. y TENDERO, G. (2015): Ja volem el pa sencer. Respostes a la pobresa alimentària en clau de Sobirania Alimentària. Barcelona, Aliança per la Soberania Alimentària de Catalunya (ASAC).

POTTER, C. (2004) Multifunctionality as an agricultural and rural concept, 15-35, in Brouwer, F. (ed) Sustaining Agriculture and the Rural Environment, Edward Elgar, Cheltenham.

RICARDO, D., (1817) On the principles of political economy and taxation. Cambridge University Press, Cambridge, 1950.

THÜNEN, J., (1826) Der Isolierte Stadt in Beziehung auf Laundwirtschaft und National o Konomze. Rostock (version original en alemán). In Peter Hall (ed.) Von Thünen's isolated state. Pergamon Press, Oxford, 1966.

TULLA, A. F., PALLARÈS-BARBERÀ, M. and VERA, A. (2009): Naturbanization and Local Development in the Mountain Areas of the Catalan Pyrenees. In Prados, M. J. (ed.): Naturbanization: New Identities and Processes for Rural-Natural Areas. London: Taylor \& Francis, p. 75-92.

TULLA, A.F., VERA, A., BADIA, A., GUIRADO, C. y VALLDEPERAS, N. (2014): «Rural and Regional Development Policies in Europe: Social Farming in the Common Strategic Framework (Horizon 2020)", Journal of Urban and Regional Analysis, Vol. VI (1), 35-52.

VERA. A.; BADIA, A.; TULLA, A., (2011) Desarrollo local en el Pirineo Catalán: Impulso económico y uso sostenible del territorio. FINISTERRA. Revista Portuguesa de Geografía, XLVI, 92: 9-27. 